\title{
Turismo gastronómico sostenible para la preservación del patrimonio culinario: las rutas del vino en Girona (Catalunya, España) y Querétaro (México)
}

\author{
Sustainable food tourism for the preservation of culinary heritage: wine \\ routes in Girona (Catalonia, Spain) and Querétaro (Mexico)
}

\author{
Marisa Morales Loya* \\ Francesc Fusté-Forné
}

Universitat de Girona, España

\section{RESUMEN}

El turismo gastronómico sostenible se desarrolla a partir de experiencias fundamentadas en el patrimonio alimentario para descubrir no solo los productos, sino también los productores y el contexto físico y humano en el que se localizan. La protección del patrimonio gastronómico y la promoción del turismo gastronómico deben realizarse siguiendo los Objetivos de Desarrollo Sostenible, contribuyendo así al desarrollo económico, ambiental, cultural y social. A partir de una investigación cualitativa comparativa, se analizan dos regiones, Girona (Catalunya, España) y Querétaro (México), que en común disponen de un producto de turismo gastronómico enfocado en el patrimonio del vino. Se entrevista a diez personas con distintos perfiles del sector vitivinícola y el turismo gastronómico de las regiones. Los tópicos de la entrevista son: (1) Rol y experiencia profesional con relación al turismo gastronómico y el sector vitivinícola, (2) Presencia e impacto del vino como patrimonio culinario de la región, (3) Acciones de desarrollo y promoción del turismo gastronómico en el sector vitivinícola, (4) Implementación del vino como producto partícipe de la sostenibilidad de la región, a través del turismo gastronómico. En los resultados se presenta la relación entre el turismo y el sector vitivinícola de las dos regiones, la producción y consumo responsable para garantizar la preservación del patrimonio y la promoción de estrategias para el desarrollo del turismo gastronómico sostenible.

Palabras clave: enoturismo, patrimonio culinario, sostenibilidad, turismo gastronómico.

Este es un artículo Open Access publicado bajo la licencia Creative Commons Atribución 4.0 Internacional (CC-BY 4.0).

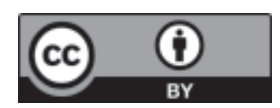

*Correspondencia: marisamoralesloya@outlook.com 


\begin{abstract}
Sustainable food tourism is developed from experiences based on food heritage to discover not only the products, but also the producers and the physical and human context in which they are located. The protection of food heritage and the promotion of food tourism must be carried out in the context of the Sustainable Development Goals, thus contributing to economic, environmental, cultural and social development. Drawing from a comparative qualitative research, two regions are analyzed, Girona (Catalonia, Spain) and Querétaro (Mexico), which have a food tourism product focused on wine heritage. Ten people with different profiles from the wine and the food tourism sector of the regions are interviewed. The topics of the interview are: (1) Role and professional experience in relation to food tourism and the wine sector, (2) Presence and impact of wine as a culinary heritage of the region, (3) Actions for the development and promotion of food tourism in the wine sector, (4) Implementation of wine as a product that participates in the sustainability of the region, through food tourism. The results present the relationship between tourism and the wine sector of the two regions, responsible production and consumption to guarantee the preservation of heritage and the promotion of strategies for the development of sustainable food tourism.
\end{abstract}

Keywords: wine tourism, food heritage, sustainability, food tourism.

\title{
Introducción
}

La presente investigación se centra en las regiones del vino de Girona (Catalunya, España) y Querétaro (México), dos regiones que comparten la producción y consumo del vino local como recurso de su gastronomía y la vertebración de una ruta del vino en el contexto del enoturismo. Asimismo, se analiza el desarrollo y promoción del turismo gastronómico sostenible y su papel para la preservación del patrimonio culinario (Santos et al., 2021).

Este trabajo contribuye en la comprensión del tema sobre la puesta en valor del vino, en el desarrollo del turismo gastronómico sostenible, al analizar la realidad de una región con una trayectoria consolidada en la elaboración y comercialización del vino y otra con una trayectoria emergente. Entre las implicaciones prácticas del estudio se encuentra la creación de sinergias entre las regiones para que a partir de las perspectivas de los agentes que participan en la elaboración y la venta, y el estudio del vino, estas consoliden el desarrollo de experiencias sostenibles a lo largo de la cadena de valor. Con relación a las implicaciones teóricas, se profundiza en estudios recientes de análisis comparativo de la región de Querétaro con respecto a la producción y comercialización de vino en Europa: la investigación de De Jesús-Contreras et al. (2020) que compara la región enológica de Querétaro y la DOP Penedès que muestra dos modelos de gestión y promoción.

Por lo tanto, se plantea como objetivo analizar y comparar el rol del turismo gastronómico sostenible para la preservación del patrimonio culinario en las rutas del vino de Girona y Querétaro. Por lo que se realiza una revisión de la literatura al respecto y se identifican acciones de desarrollo y promoción enfocadas a la sostenibilidad a partir de la cadena de valor del turismo gastronómico. Además, se presenta información propia 
del sector vitivinícola de las mencionadas regiones que contribuye con la valoración turística de la tradición vitivinícola, a partir de la opinión de los agentes implicados en su protección y promoción.

\section{Sostenibilidad, turismo y gastronomía}

De acuerdo a la Organización Mundial del Turismo (OMT, 2021) el desarrollo sostenible en el turismo se refiere al «turismo que tiene plenamente en cuenta las repercusiones actuales y futuras, económicas, sociales y medioambientales para satisfacer las necesidades de los visitantes, de la industria, del entorno y de las comunidades anfitrionas». Para lograrlo, los agentes de turismo, deben trabajar en la creación de sinergias colaborativas de trabajo para el intercambio de conocimientos y experiencias sobre las motivaciones y efectos del sector turístico en los turistas, el destino y las empresas, basándose en la perspectiva de los Objetivos de Desarrollo Sostenible (Azcárate et al., 2019). En este cometido los destinos deben asegurar la existencia de los principios de la sostenibilidad y sus variables socioeconómicas, ambientales y culturales, así como la implicación de las empresas que conforman el sector turístico y de los propios turistas. El desarrollo sostenible del turismo requiere la participación informada de todos los agentes que conforman el sector turístico del ámbito público y privado; el éxito de un turismo sostenible depende de un proceso continuado con seguimiento constante de los impactos (Torres, 2016). Además, se requiere de una gestión sostenible eficaz de los destinos para así minimizar los efectos adversos que puedan generarse y se transforme el turismo en un sector que contribuya a los ODS (OMT y BCC, 2019; OMT y OEA, 2018).

Sin embargo, por la dualidad de las consecuencias del turismo, un crecimiento no planificado del sector conduce al deterioro de los recursos. Ante esta situación, en los destinos se ha optado por ofrecer experiencias auténticas y sostenibles en contacto con el medioambiente y la comunidad, debidamente reguladas por normativas que incorporan la gestión pública y privada; basadas en la protección del patrimonio cultural y natural (OMT, 2010). El ecoturismo o el turismo rural son ejemplos de esta línea de gestión. El ecoturismo incrementa la sensibilización respecto a la conservación de los ecosistemas locales. La OMT (2019) define ecoturismo:

Tipo de actividad turística basada en la naturaleza en el que la motivación esencial del visitante es observar, aprender, descubrir, experimentar y apreciar la diversidad biológica y cultural, con una actitud responsable, para proteger la integridad del ecosistema y fomentar el bienestar de la comunidad local. (p. 34)

Mientras que, el turismo rural, según la OMT (2019) es un «tipo de actividad turística en el que la experiencia del visitante está relacionada con un amplio espectro de productos vinculados por lo general con las actividades de naturaleza, la agricultura, las formas de vida y las culturas rurales» (p. 35). Las actividades de turismo rural se realizan en entornos periféricos que suelen ofrecer una baja densidad demográfica y una estructura económica que tradicionalmente se ha basado en las actividades del sector primario.

En cuanto al turismo sostenible, la OMT (2019) destaca que la sostenibilidad no es un atributo de un tipo de turismo; vale decir, cualquier desarrollo turístico sea tradicional o alternativo, como por ejemplo el ecoturismo, debe manejarse con criterios sostenibles. El turismo es una actividad económica dependiente del territorio y la 
calidad de sus atractivos que representan su permanencia a largo plazo. Sidorenko y Garrido (2021) identifican el turismo sostenible como un modelo enfocado al desarrollo económico, social y medioambiental de un lugar y destacan que su crecimiento sucede el doble de rápido que el del turismo convencional. Pérez (2004), complementa, señalando que el objetivo fundamental del turismo sostenible es mantenerse en el tiempo, protegiendo los recursos que lo sostienen, respetando e involucrando a la comunidad.

Por otra parte, la gastronomía es «un área interdisciplinar que estudia y genera procesos físico-químicos, culturales y socioeconómicos donde el ser humano cultiva, procesa, distribuye y consume buenos alimentos y bebidas que afectan su bienestar físico, mental y social» (OMT y BCC, 2019, p. 8). La gastronomía ha formado siempre parte del turismo, sin embargo, en las últimas décadas ha tenido un cambio significativo, despertando mayor interés como un factor de atracción turística (Ellis et al., 2018). Para Fusté-Forné (2015) mediante la gastronomía se integran los rasgos característicos del paisaje natural y cultural del destino. En consecuencia, el papel de la gastronomía en la definición y en la diferenciación de la oferta de los destinos turísticos es cada vez más relevante a nivel internacional (Okumus, 2020). Asimismo, se han implementado nuevos modelos de actividades, abriendo un nuevo segmento turístico, conocido como turismo gastronómico (Hall y Sharples, 2003; Henderson, 2009; Fusté-Forné, 2020).

El turismo gastronómico, según la OMT (2019), es una actividad turística para que el viajero tenga vivencias y disfrute de productos relacionados con la gastronomía de un lugar; por medio de los restaurantes, las visitas a productores locales, la participación en festivales gastronómicos o en clases de cocina. Se basa, el turismo gastronómico, en descubrir la cultura gastronómica relacionada con un territorio y sus costumbres, por lo que hablar de gastronomía es hablar de la identidad culinaria de un destino (Kim y Iwashita, 2016; Long, 2004). Este turismo integra a todos los actores de la cadena productiva de la alimentación y, además, contribuye al desarrollo sostenible de los destinos por medio de la producción y consumo responsables. Al ser una actividad transversal, maximiza la distribución de beneficios del turismo en las comunidades y empodera a todos los participantes de la cadena de valor gastronómica, lo que refuerza la identidad y el sentido de pertenencia para así resguardar la autenticidad del lugar (OMT y BCC, 2019).

Respecto del turismo gastronómico sostenible, Velásquez y Garlobo (2020), consideran que se realiza por medio de actividades fundamentadas en el patrimonio gastronómico material e inmaterial en lugares diferentes al entorno habitual, con el principal objetivo de realizar experiencias gastronómicas donde se valoren los productos, la sociedad y su cultura, así como el uso responsable de los recursos naturales. Es decir, que los alimentos se empleen de manera saludable, cultivados y producidos con responsabilidad ambiental y promocionados dentro de la gastronomía tradicional por medio de actividades sostenibles que contribuyan a mantener la identidad del patrimonio gastronómico. Torres (2016), por su parte, relaciona al turismo gastronómico sostenible con la creación de un sistema de seguridad alimentaria regional donde se tome en cuenta la solución a problemáticas como hambre, desnutrición, malbaratamiento y desperdicio. En síntesis, el turismo gastronómico sostenible es la unión de la práctica del turismo gastronómico y el desarrollo de estrategias basadas en la preservación del territorio por medio del cuidado medioambiental, de aspectos culturales y de patrimonio, de aspectos socioculturales y de la economía del destino para beneficio de los residentes y sus actividades (Hjalager y Richards, 2003). 
Con relación a lo planteado, se encuentra el enoturismo que es «un subtipo del turismo gastronómico, se refiere al turismo cuya motivación es visitar viñedos y bodegas, realizar catas, consumir y comprar vino, a menudo en el lugar en que se produce o en sus cercanías» (OMT, 2019, p. 41). Como se viene desarrollando, se puede considerar como una tipología turística independiente; su objetivo es visitar y realizar paseos por los viñedos, aprender el proceso de elaboración del vino, degustar vinos, visitar museos o actividades de cuidado corporal como masajes con productos hechos a partir de las pieles de las uvas (Molleví y Fusté-Forné, 2016). Dentro del enoturismo destacan las rutas del vino que son rutas gastronómicas tematizadas y con larga historia de desarrollo en Europa (Charters y Menival, 2011) y otras partes del mundo como Estados Unidos (Wargenau y Che, 2006), Australia (Carlsen y Dowling, 1998) y Nueva Zelanda (Alonso, 2009). En Europa, las rutas fueron promocionadas principalmente para conocer el patrimonio cultural donde se visita, en grupos, atractivos de un destino con la colaboración de agentes públicos y privados (Jeambey, 2016). Una ruta del vino es un producto turístico complejo que es el resultado de una red de cooperación empresarial (Romero, 2017). Molleví y Fusté-Forné (2016) mencionan que las rutas son una oportunidad para aumentar las actividades turísticas en territorios del interior, lo cual ayuda a la reducción de la estacionalidad turística.

Para el sector turístico, el turismo gastronómico tiene gran importancia debido a que su característica principal, el patrimonio culinario, es el factor que diferencia a los destinos (López-Guzmán y Sánchez-Cañizares, 2012; Velásquez y Garlobo, 2020); y también los posiciona (Du Rand y Heath, 2006; Rousta y Jamshidi, 2020). El turismo gastronómico, bien gestionado, tomando en cuenta los Objetivos de Desarrollo Sostenible tiene la capacidad de contribuir al desarrollo social y al crecimiento económico, a partir de la producción y consumo responsables lo que beneficia la preservación del patrimonio culinario (OMT y BCC, 2019).

\section{Empordà y Girona (Catalunya, España)}

Empordà se ubica geográficamente en la zona nororiental de Catalunya, entre la zona de los Pirineos y el Mar Mediterráneo. Catalunya es un destino de turismo gastronómico por su tradición culinaria, gracias a las diversas culturas y civilizaciones que han formado parte de su historia y que ha heredado la tradición de productos como el vino (Jeambey, 2016). La cultura del vino y el conocimiento de la viña llega a Cataluña en el siglo VI a. C. gracias a la colonia griega de Empúries, ciudad que dará nombre a la comarca del Empordà. La producción de vino se consolida progresivamente a partir de la Edad Media hasta la plaga de la filoxera en la segunda mitad del siglo XIX. Después, existe un resurgimiento de la producción vitivinícola que inicia el movimiento de las bodegas cooperativas en la primera mitad del siglo XX. Específicamente en la región del Empordà, en 1975 se aprueba la Denominación de Origen Protegida Empordà-Costa Brava (Consell Regulador de la Denominació d’Origen Protegida, 2021).

Dentro del enoturismo en Girona se encuentra la Ruta del Vino DOP (Denominación de Origen Empordà), una experiencia en torno al vino de la región y los paisajes culturales y naturales que forman su identidad. La zona de producción engloba 55 municipios distribuidos en las comarcas del Alt Empordà (35 municipios) y del Baix Empordà (20 municipios). La DOP Empordà cuenta con 2000 hectáreas de viñas, con 268 viticultores y 51 bodegas (Consell Regulador de la Denominació d’Origen Protegida, 2021). En la zona se cultivan variedades de uvas blancas y tintas, dentro de las que predominan como blancas la Garnacha blanca, Macabeo y Moscatel 
de Alejandría. En cuanto a las variedades tintas, predominan la Garnacha tinta, Samsó y Cabernet Sauvignon. En cuanto a su producción, esta ronda entre los 65000 hectolitros que representan 6 millones de botellas anuales. En relación con el turismo, la Ruta del Vino DOP Empordà en 2019 fue visitada por más de 56000 personas, de las cuales $64 \%$ son procedentes de Cataluña y 36\% son visitantes nacionales o internacionales (La Costa Brava promociona su enoturismo con una campaña, 2020).

\section{Querétaro (México)}

El estado de Querétaro se ubica en la región centro norte de la República Mexicana y cuenta con una zona vitivinícola ubicada en 11 de sus 18 municipios: Querétaro, Amealco, Cadereyta de Montes, Colón, El Marqués, Ezequiel Montes, Tolimán, San Juan del Río, Tequisquiapan, Pedro Escobedo y Huimilpan. El enoturismo representa una manera atractiva de conocer estos lugares; una de las estrategias del Gobierno Estatal es la promoción de la ruta Arte, Queso y Vino, creada en 2016 en coordinación con las Secretarías de Turismo y de Cultura. Se abarcan tres áreas fundamentales representativas de las riquezas de Querétaro, para ello, se integra el vino como producto de la región, se le denomina: La Ruta del Vino. La vitivinicultura queretana proviene de la época colonial durante el establecimiento de las primeras comunidades novohispanas y la fundación de misiones religiosas. En 1554 se plantaron los primeros viñedos por los misioneros para elaboración de vinos sacramentales. Entre 1600 y 1700 se asoció a la viticultura con actividades desarrolladas en haciendas y entre 1917 y 1940 se posicionó como actividad de ranchos. El surgimiento del enoturismo en el territorio, así como el máximo esplendor de la viticultura queretana comprende entre 1950 y 1985 con el crecimiento de aproximadamente 3500 hectáreas. La apuesta por el turismo se consolida en 2007 con la implementación de la primera versión de la Ruta del Vino (Contreras et al., 2019).

Es considerada como la región ubicada más al sur del hemisferio norte en el mundo, caracterizándose así por una viticultura extrema (Asociación de vinicultores de Querétaro, 2020). En Querétaro se encuentra una de las viticulturas más prósperas del país por sus características geoclimáticas (Arévalo, 2018). La región cuenta con 500 hectáreas de vid plantadas, un rendimiento promedio de 10 toneladas por hectárea y una producción de 5000 toneladas anuales (Contreras et al., 2019). Existen más de 30 bodegas cuya producción representa más de 200 etiquetas con una producción de 3.5 millones de botellas anuales. Las principales variedades de uva tinta son Merlot, Syrah, Malbec, Marselan, Tempranillo y Cabernet Sauvignon; como uva blanca se encuentran Macabeo, Xarel·lo, Chardonnay y Sauvignon Blanc (Asociación de Vinicultores de Querétaro, 2020). La Ruta del Vino de Querétaro, de acuerdo a la Secretaria de Turismo del Estado de Querétaro (SECTUR, 2019), representa el segundo producto turístico más importante de la región, con un promedio de 1 millón de visitantes anuales.

\section{Metodología}

Esta es una investigación cualitativa comparativa. Se realiza estudio de caso de dos regiones, Girona y Querétaro, que en común disponen de un producto de turismo gastronómico enfocado aen el patrimonio del vino. La técnica utilizada es la entrevista; a partir de un muestreo de conveniencia no probabilístico se selecciona a diez personas, cinco de cada región, con distintos roles dentro de las actividades relacionadas con el sector 
vitivinícola y el turismo gastronómico de las dos regiones (Tabla 1). La entrevista aborda cuatro tópicos: (1) Rol y experiencia profesional con relación al turismo gastronómico y el sector vitivinícola, (2) Presencia e impacto del vino como patrimonio culinario de la región, (3) Acciones de desarrollo y promoción del turismo gastronómico en el sector vitivinícola, (4) Implementación del vino como producto partícipe de la sostenibilidad de la región, a través del turismo gastronómico. Debido a la situación de confinamiento por la pandemia de la COVID-19, los participantes fueron contactados mediante aplicaciones electrónicas para hacer las entrevistas en línea, las cuales se efectuaron durante los meses de abril, mayo y junio de 2021, la duración promedio de cada entrevista fue de cuarenta minutos y todas se grabaron para posterior análisis de las respuestas.

Tabla 1

Perfiles de entrevistados

\begin{tabular}{cllcl}
\hline N. ${ }^{\circ}$ & Región & Género & Edad & Rol \\
\hline 1 & Girona & Masculino & 40 & Sommelier \\
2 & Girona & Masculino & 48 & Coordinador educativo \\
3 & Girona & Masculino & 28 & Sommelier \\
4 & Girona & Femenino & 45 & Empresaria del sector y restauradora \\
5 & Girona & Masculino & 39 & Empresario del sector \\
6 & Querétaro & Masculino & 37 & Empresario del sector \\
7 & Querétaro & Femenino & 24 & Sommelier \\
8 & Querétaro & Masculino & 32 & Empresario del sector \\
9 & Querétaro & Masculino & 29 & Restaurador \\
10 & Querétaro & Femenino & 33 & Directora educativa \\
\hline
\end{tabular}

\section{Resultados}

Los resultados corresponden a los temas abordados mediante las entrevistas: (1) Rol y experiencia profesional con relación al turismo gastronómico y el sector vitivinícola, (2) Presencia e impacto del vino como patrimonio culinario de la región, (3) Acciones de desarrollo y promoción del turismo gastronómico en el sector vitivinícola, (4) Implementación del vino como producto partícipe de la sostenibilidad de la región, a través del turismo gastronómico. Se detallan las prácticas de desarrollo sostenible y promoción del sector de turismo gastronómico relacionado con el sector vitivinícola de las regiones de Girona y Querétaro, considerando el producto del vino como patrimonio culinario. En la Tabla 2 se presentan algunos extractos de las entrevistas efectuadas a los 10 participantes en la investigación; quienes se desempeñan en roles diversos: rol de propietario de vitivinícola, rol de personal relacionado con la restauración, rol de consultores y académicos. 
Tabla 2

Ejemplos de citas de las personas entrevistadas

Tema Ejemplos

1 «Es importante que todo el equipo esté formado para saber explicar el vino, para que lo conozcan, para que puedan hablar de él. Conoces un vino cuando sabes quién lo hace realmente y ves de dónde ha salido la tierra y la persona» (entrevistado 1).

«Donde más trabajo tengo es la gente que quiere visitar nuestras viñas y yo lo que hago es explicar la filosofía del viticultor y lo que hacemos en el campo, lo que hacemos en la bodega» (entrevistado 5).

«Optamos por incluir en nuestra oferta académica un curso de enoturismo como para dar a conocer el auténtico enoturismo, un enoturismo sustentable, un enoturismo local, las buenas prácticas de enoturismo también de hospitalidad» (entrevistado 10).

2 «Los sommeliers de la región han hecho mucho trabajo a la hora de potenciar y de enseñar los vinos de la zona, que la gente los conozca y actualmente mucha gente ya al ver la carta de vino te dice que quieren un vino de aquí» (entrevistado 1 ).

«No se entiende la historia gastronómica cultural sin los vinos del Empordà» (entrevistado 3).

«Hay una apropiación del vino, pero yo creo que nos va a tomar tiempo llegar ... tenemos la suficiente historia para poder hacerlo parte del patrimonio gastronómico, pero ha tenido altibajos por lo que gran parte de este tiempo no ha estado muy presente en la mente del consumidor» (entrevista 6).

3 «El consumo del vino es una actividad turística sostenible, porque lo que hace es acercar al visitante al origen del producto que en muchas ocasiones son entornos rurales, fomenta el conocimiento de la producción del vino, de las variedades endógenas del territorio, que la gente gaste y compre producto local, que por supuesto no sólo es beber, también comer. La gente a la vez que compramos vino, podemos comprar otros productos, compramos queso, aceite, embutidos» (entrevistado 2).

«Veo que el consumo va muy arraigado a ciertas marcas o ciertos sellos que den una connotación de seguridad o de calidad entonces para mí en Girona el consumo de vino de producción local es más con sello que sin sello» (entrevistado 3).

«En una región donde hay más historia, tiene más superficie plantada ... sí creo que la Denominación de Origen agregue ciertos valores, pero también hay ciertas limitaciones...» (entrevistado 6).

«En una región como Girona sí ... una denominación significa control en la producción ... hemos hablado de esto con los productores y estamos convencidos que es algo que por el momento no nos conviene, porque somos una región muy joven ...nos faltan años de madurez que únicamente vamos a alcanzar con el estudio» (entrevistado 10).

4 «A la larga cuando produces el vino, al final estás dando vida a mucha gente ya no solo al que puedes comprar la uva o al que te controla la viña sino hay muchos sectores que buscan hacerlo de forma sostenible, hay mucha conciencia» (entrevistado 1 ).

«Si hay inversiones de parte de las administraciones públicas para el desarrollo del turismo del vino, que estas inversiones en primer lugar tengan una perspectiva de sostenibilidad, tengan una perspectiva de respeto a los residentes y no sean meramente de promoción y de captación de visitante a cualquier precio» (entrevistado 2).

«Somos una región productora muy joven, por lo cual apenas se está formando la personalidad de la región. Es una región liderada por generaciones mayores ... creo que necesitamos dar un brinco generacional, para poder apostar por una economía sustentable» (entrevistado 10). 


\section{Rol y experiencia profesional con relación al turismo gastronómico y el sector vitivinícola}

Los roles de las personas entrevistadas se replican en las dos regiones del estudio. Se encuentra el rol de propietario de vitivinícola; la particularidad de este grupo es el vínculo con el sector por herencia familiar y son considerados los principales conocedores y productores del vino. Seguido, se cuenta con el rol de personal relacionado con la restauración; son los responsables de dar a conocer las propiedades del vino y de transmitir la sensibilidad, características y antecedentes que envuelven a los comensales, promoviendo así el patrimonio cultural de las productoras de vino. Luego, el rol de consultores y académicos, quienes asesoran dependencias relacionadas a la promoción del vino por intermedio del turismo gastronómico. Se encuentran diferencias en aspectos específicos sobre lo que ofrecen, pero ambas regiones, se enfocan en la especialización del sector como parte importante del desarrollo del mismo.

Dentro de estos roles se comparten las actividades que crean un vínculo estrecho con el sector vitivinícola y promueven el conocimiento en torno al vino, su producción y sus propiedades. En los restaurantes se ofrece la experiencia de maridaje de alimentos locales con vinos locales, resaltando el interés en la existencia del complemento entre productos y dar a conocer la gastronomía representativa. Las vitivinícolas se relacionan con el sector turístico por las experiencias de las visitas, el conocimiento del proceso, la historia de la producción y la elaboración de cada vino. En las escuelas el interés es por la especialización en el producto del vino y la relación entre el sector agroalimentario y el turismo, por parte de los estudiantes y de las instituciones, al igual que su facilidad para crear alianzas con el sector privado y público para complemento de orientación.

Respecto a los productos ofrecidos por el sector vitivinícola, se hace la distinción que, al formar parte del turismo gastronómico no solo existe el producto del vino, sino que a este se le suman actividades que se consideran como servicios que se ofrecen en el sector. Las vitivinícolas en ambas regiones organizan servicios de visitas, catas, maridajes y eventos como parte de las vitivinícolas, además de la venta del vino. En la restauración se ofrecen cartas de vino para consumo in situ, pero también para catas y maridajes. En el sector académico, comparten como producto los cursos y talleres ofrecidos. Y en el sector de consultoría, por parte de asociaciones, los servicios de orientación, vinculación y promoción para los productores de la región. Cada servicio conserva propuestas de valor enfocados en los aspectos de calidad, coherencia con su filosofía, personal especializado, proveedores con iniciativas afines, actualización de conocimientos y pertenencia a una región reconocida respecto a la producción del vino.

\section{Presencia e impacto del vino como patrimonio culinario de la región}

Con relación al crecimiento de la producción del vino y del enoturismo, existen diferencias entre ambas regiones, siendo Girona una región de mayor terreno y tiempo con presencia de vino, a diferencia de Querétaro que es considerada una región joven y en actual crecimiento. Las rutas del vino de cada región poseen particularidades, sin embargo, el impacto de contar con una ruta del vino es positivo en ambas, debido al enfoque que se brinda del producto y la promoción del sector vitivinícola, así como por la atracción de visitantes que se convierten en consumidores potenciales mediante sinergias colaborativas entre el sector de restauración y el sector vitivinícola. Las ventajas de este tipo de rutas son el posicionamiento de los productores en el mercado 
del vino, la creación de productos competitivos, la diversificación de economías y servicios, y la colaboración entre el sector público y privado, permitiendo el desarrollo y sostenibilidad de la región. Paralelo a esto, como inconvenientes, se identifica la inversión en recursos de promoción turística requerida por parte de los productores que en ocasiones no forma parte de su presupuesto y la incertidumbre en el funcionamiento adecuado de la ruta de acuerdo a los propósitos de la misma y la participación de varios actores.

La percepción de la trayectoria en el consumo turístico del vino en cada región se enfoca en aspectos como el tiempo de existencia del sector vitivinícola, evidenciando un crecimiento del mismo, la existencia de una falta de conocimiento del vino fuera de la región donde se produce y en el interés de conocer el vino por los visitantes ya que es un ejemplo representativo de los paisajes gastronómicos. En Girona se percibe un consumo que se ha incrementado a la par del crecimiento de la región debido a la promoción por parte de profesionales conocedores del vino y de eventos realizados que apuestan por lo local y que han diversificado su presencia en el sector gastronómico. En Querétaro se percibe que el consumo ha crecido por el conocimiento de la ruta del vino; sin embargo, también se percibe falta de identificación con el vino en la región, así como estabilidad de crecimiento en el ámbito enogastronómico como región que vincule la gastronomía con el sector vitivinícola. En cuanto al perfil del consumidor de la ruta del vino, en Girona se encuentra un rango de edad que va de 30 a 60 años, en su mayoría locales, tomando en cuenta visitantes franceses por la cercanía a la frontera. En Querétaro, el rango de edad es entre 25 y 45 años, en su mayoría visitantes locales y nacionales. Toman en cuenta el factor económico como parte decisiva en el consumo.

La existencia de una apropiación de vino como parte del patrimonio culinario en cada región se considera de forma diferente. En Girona coinciden en que el vino forma parte de su patrimonio culinario, por diversos factores como contar con variedades de uva tradicionales, el vino como parte de la cultura que envuelve la historia de la región y que se incluye en la gastronomía de la región. En Querétaro la mayoría coincide en que forma parte del patrimonio culinario, sin embargo, es una región que aún no dispone de una cultura vitivinícola arraigada para identificarse como patrimonio. No se descarta que exista esta distinción más adelante cuando se genere mayor presencia en el consumo, implementación de especialistas y formación académica en el tema. Por lo tanto, se puede determinar en ambas visiones que el vino pertenece al patrimonio culinario, pero se necesita trayectoria y trabajo para ser reconocido así de parte del consumidor y la región misma; así como para desarrollar su atractivo turístico.

\section{Acciones de desarrollo y promoción del turismo gastronómico en el sector vitivinícola}

Los productos y servicios relacionados con el sector vitivinícola, importantes para la conservación del turismo gastronómico, son las actividades de difusión y recreación de las vitivinícolas, los productos gastronómicos que complementan el consumo del vino, los servicios de hostelería y restauración en la región vitivinícola o en municipios cercanos, los eventos y ferias enfocados a la promoción del vino y los medios de transporte que faciliten el acercamiento, lo cual muestra la relación entre los diferentes aspectos del sistema turístico. Relacionando estos productos y servicios con el desarrollo sostenible en la región, el sector vitivinícola debe ser parte de la promoción del desarrollo de la comunidad por la diversidad de acciones que se ofrecen en torno al mismo, el acercamiento con el origen del producto, y por fomentar el conocimiento y el consumo de 
productos locales. En este sentido, es necesario el aprovechamiento sostenible de los recursos locales, la responsabilidad en el proceso de elaboración y la promoción de todos los involucrados en el sector.

En cuanto a las acciones de desarrollo y promoción del turismo gastronómico que contribuyen a la preservación del patrimonio culinario, se consideran primordialmente a la propia ruta de vino implementada en cada región y, a partir de estas, acciones específicas.

Primero, acciones de desarrollo como contar con una planeación estratégica de la región referente a turismo gastronómico, crear acciones colaborativas entre el sector privado y público de los diferentes rangos de administración, así como un acercamiento con asociaciones representativas del sector vitivinícola. Luego, realizar actividades con otros productores agroalimentarios pertenecientes a la región, crear esfuerzos para trazar un precio accesible al producto, generar concientización del vino como parte de la historia, cultura y representación de la región y posteriormente desarrollar planes de control y supervisión que aseguren la competitividad del sector. Además, las regiones deben disponer de responsables del sector turístico especializados y capacitación y formación académica a productores y promotores del sector de enoturismo.

Segundo, acciones de promoción como la vinculación entre el sector vitivinícola y de restauración local para la promoción de productos que fomenten en conjunto la gastronomía representativa de la región, su identidad cultural y natural. A la vez, una estrategia de promoción en medios de comunicación que destaque al vino como bebida parte de la gastronomía de la región, la venta y recomendación de productos por medio de especialistas en el tema del vino y la organización de eventos sociales y culturales para la atracción de visitantes a la región e implementación de medios de publicidad que fomenten un conocimiento claro de los proyectos actuales de turismo gastronómico vinculados al vino. En estas acciones se puede observar la orientación a realzar las características de la región para reconocer al vino como parte de la gastronomía, tomar conciencia de la importancia para la localidad y la unión de esfuerzos necesaria para lograr el acercamiento al conocimiento de la bebida. Igualmente, se puede observar que el sector vitivinícola de las regiones Empordà y Querétaro participa en la cadena de valor del turismo gastronómico.

Una parte que se distingue dentro del sector vitivinícola es la implementación de la Denominación de Origen Protegida (DOP), establecida en regiones que poseen variedades de uvas representativas y que se han regulado para la producción. En este caso, el estudio contempla una región que no tiene aún nombramiento que la represente y una región que cuenta con este nombramiento, Denominación de Origen Protegida Empordà, en Girona. En este planteamiento se encuentra que parte de la especialización en torno al conocimiento del vino y su producción, adquirida con el tiempo, permite disponer de una DOP. Sin embargo, en regiones sin tradición vitivinícola no es viable porque no se tiene la estabilidad y experiencia requerida. En Girona la DOP es parte de la característica del sector vitivinícola en la región y culturalmente forma parte de la misma, igualmente se vincula a la calidad y seguridad del producto. En Querétaro se reconoce que en regiones con mayor impacto del sector vitivinícola la etiqueta de calidad fomenta el consumo pero en la región queretana, al ser más reciente en el tema, se requiere crecimiento, experiencia en el control del cultivo respecto a las variantes del entorno y estabilidad. Actualmente una DOP limitaría la experimentación y producción de nuevos vinos, así como el tipo de variedad plantada; a pesar de ello, se observa la idea de poder implementar otro tipo de certificación que 
se adapte a las regulaciones de México, conocimiento de esta distinción por parte de los residentes e involucrados en el sector. Las ventajas de la DOP se basan en el valor agregado a la región y la confianza que transmite el producto por la calidad y seguridad adquirida por una producción regulada; no obstante, los inconvenientes encontrados son mayores debido a que requiere de una mayor tradición vitivinícola para un impacto en el consumo, a la vez que se limitan las variedades y métodos de producción y la experimentación e innovación de nuevos productos e ingreso de nuevos productores al mercado.

\section{Implementación del vino como producto partícipe de la sostenibilidad de la región, a través del turismo gastronómico}

A partir de las entrevistas realizadas, las acciones de producción y promoción del vino dentro de la cadena de valor del turismo gastronómico que contribuyen a la sostenibilidad de la comunidad local, se clasifican de acuerdo a factores como la estructura de las vitivinícolas, donde se plantea implementar nuevos modelos de cultivo, tener una producción ecológica con infraestructura e instalaciones que permitan practicar la sostenibilidad. Así, es necesaria la aplicación de prácticas medioambientales como el desarrollo de una infraestructura con herramientas de transformación de energía, la realización de estudios de impacto ambiental previos a la plantación de un viñedo y regulaciones por medio de certificaciones que aseguren buenas prácticas en la producción de vino. A la vez, se requiere del fomento de empleo de la población local en los servicios del sector vitivinícola y turístico, y la promoción de consumo local del vino, igual que el acercamiento al sector vitivinícola y sus recursos por medio de la creación de actividades que involucren el conocimiento del producto para posterior identificación y crear una fidelización entre el visitante, la región y sus productos. Se precisa una especialización académica en temas relacionados con la vitivinicultura que transmitan correctamente los productos del sector y sus valores. Por último, es importante generar colaboraciones entre el sector vitivinícola y el sector de la restauración para resaltar la gastronomía queretana con los productores de la región para mayor conocimiento del vino y con administraciones públicas para inversión, promoción y regulación de prácticas sostenibles enfocadas en la región vitivinícola.

Posterior al conocimiento de estas acciones de sostenibilidad se afirma que el vino puede ser parte de la sostenibilidad de la región mediante el turismo gastronómico, realizándose por medio de una planeación estratégica conforme a acciones en torno al uso de recursos locales durante la producción y promoción del vino, igualmente, mediante acuerdos y la inversión de capital para la implementación de prácticas sostenibles. Los beneficios que se encuentran al promover la sostenibilidad en la producción y promoción del vino son el desarrollo de la región y la apropiación del vino como parte de la economía local, de la sociedad y la cultura.

Para posicionar al vino dentro del turismo gastronómico sostenible se requiere la participación de actores clave de los tres sectores económicos. La sostenibilidad del turismo gastronómico debe partir también de las relaciones responsables entre los productores, elaboradores de vino, actores de comercialización integrados en el sistema turístico. En el sector público se precisa de la coordinación entre gobiernos locales y regionales para regular en prácticas sostenibles, vigilar el accionar de las instituciones públicas y destinar presupuestos a proyectos sostenibles. A la vez, es necesaria la colaboración con el patronato o secretaría de turismo que unifique y relance proyectos de promoción del sector vitivinícola y con entes de desarrollo social que trabajen con productores de la región. 
En el sector privado se requiere de vitivinícolas que implementen prácticas sostenibles, que la restauración proteja y promocione proyectos de consumo local; escuelas con formación en temas de sostenibilidad en el sector; empresas certificadoras que otorguen y vigilen el cumplimiento de las prácticas realizadas; líderes de opinión que promuevan la importancia del sector y su sostenibilidad; y que los comerciantes aseguren procesos conforme a la sostenibilidad e informen a los consumidores. Asimismo, se requiere del tercer sector conformado por asociaciones de vitivinicultores, del sector de restauración y de perspectiva social, que conecten y orienten a los vitivinicultores con proyectos sostenibles y con perspectiva social.

\section{Conclusiones}

En esta investigación se analiza la promoción y desarrollo del turismo gastronómico sostenible de las rutas del vino de Girona (Catalunya, España) y Querétaro (México), así como la preservación del patrimonio culinario, en este caso, el vino. A partir de los resultados se observa la presencia del turismo gastronómico relacionado con el sector vitivinícola en actividades directas, como las visitas a las vitivinícolas y colaboraciones con asociaciones pertenecientes al sector y la especialización en instituciones académicas. Se destaca en ambas regiones la realización de actividades pertenecientes a los diferentes procesos de la cadena de valor del turismo gastronómico (Hjalager y Richards, 2003); indicando que existe un interés en la implementación de servicios desde la producción hasta la formación académica. Cada región cuenta con características propias, que las distinguen, pero también tienen similitudes en la producción del vino y la promoción del sector en el contexto del turismo gastronómico de la región. Por ejemplo, mediante las rutas del vino como un factor de atracción turística que incentiva la colaboración entre sectores para el desarrollo de la región.

La apropiación del vino como parte del patrimonio culinario depende de los planes trazados previamente en la trayectoria de la región; de esta manera logra ser identificada y reconocida como una región enoturística. En ambos casos se determina esta identificación debido a que el vino es un producto que forma parte de la gastronomía y producción local, quedando solo formar una consolidación para ser reconocido más ampliamente por los consumidores. Es decir, la cultura alimentaria pasa a ser digna de patrimonialización conforme al crecimiento del patrimonio cultural e inmaterial (Medina, 2017). En este sentido, el hecho de reconocer la producción mediante una DOP representa una característica diferenciadora en regiones como Girona que han establecido una protección y promoción del sector vitivinícola, que evidencia en su trayectoria e historia, mientras que en regiones donde se está recientemente estableciendo el valor del sector, como Querétaro, sería un factor que limitaría el progreso del enoturismo debido a que se encuentran en etapas de experimentación e instauración de métodos y procesos.

Respecto al desarrollo del turismo gastronómico, los resultados muestran la finalidad de la conservación del patrimonio culinario, mediante la participación colaborativa para la promoción del conocimiento del sector y el uso responsable de recursos locales. Estas son acciones que la OMT y BCC (2019) recomiendan como estrategias para la conformación del turismo gastronómico sostenible. La aportación del turismo gastronómico hacia el desarrollo sostenible de la región se representa en la cadena de valor del turismo gastronómico orientado al desarrollo social, económico y medioambiental del sector vitivinícola. Para cumplirlas es necesaria la participación de los tres sectores en estrecha colaboración enfocados en la sostenibilidad y siguiendo los ODS. 
La idea de la preservación del patrimonio culinario mediante el turismo gastronómico sostenible se observa como viable si se aplican estrategias de desarrollo y promoción orientadas al desarrollo social. Un factor importante, parte de esta preservación, además del desarrollo y promoción de estrategias, es la concientización referente a la sostenibilidad y al valor del patrimonio gastronómico, fungiéndolos como motores de desarrollo social que permiten la preservación de la cultura y, por lo tanto, de los productos que integran esta cultura. El turismo gastronómico sostenible es el turismo gastronómico con estrategias que se basan en la preservación del patrimonio cultural y natural y de la proyección sostenible de la economía del destino para beneficio de los residentes y sus actividades. Para ello, se requiere, además de la definición, la comprensión de la sostenibilidad dentro del turismo gastronómico. La característica de sostenibilidad, como señala la OMT (2019) debería ser inherente en el desarrollo de cualquier tipo de turismo, sin la necesidad de realizar el nombramiento, por lo que es necesario la práctica del mismo en cualquier tipología de turismo debido a la relación directa con el entorno, la población y sus recursos.

Nuevas investigaciones relacionadas con el tema pueden dar continuidad a la idea de la preservación del patrimonio culinario mediante el turismo gastronómico realizado a partir de los ODS, como el conocer estadísticamente a partir de la demanda su apreciación del sector y del patrimonio culinario. Es pertinente mencionar el reconocimiento del cambio en actividades y consumo a partir de la pandemia de la COVID-19, que está generando no solo retos a combatir en el ámbito vitivinícola, sino la posibilidad de una orientación del sector durante la recuperación, siendo la sostenibilidad una característica sine qua non en el desarrollo de experiencias del turismo gastronómico.

\section{Agradecimientos}

A Fundación Carolina, el Patronat de Turisme Costa Brava Pirineu de Girona, la Càtedra de Gastronomía, Cultura i Turisme Calonge - Sant Antoni y la Universitat de Girona por los estudios de maestría en Turismo Cultural mediante la beca de formación otorgada a la primera autora de este artículo.

\section{Conflicto de intereses}

Los autores no tienen conflicto de intereses con el contenido de este trabajo.

\section{Contribución de autoría}

MML: Conceptualización, metodología, adquisición de datos, análisis de datos y redacción del artículo. FFF: Conceptualización, metodología, análisis de datos, redacción del artículo y revisión final del artículo. 


\section{Referencias}

Alonso, A. D. (2009). Are travellers interested in wine tourism in New Zealand? International Journal of Culture, Tourism and Hospitality Research, 3(1), 13-24.

Arévalo, G. (2018). La ruta turística enológica en Querétaro y Baja California, México: Un enfoque estratégico. Revista Interamericana de Ambiente y Turismo, 14(2), 122-134.

Asociación de Vinicultores de Querétaro. (2020). Vinos y viñedos de Querétaro. https://www.avq.com.mx/index.html

Azcárate, T., Benayas, J., Nerilli, G. y Justel, A. (2019). Guía para un turismo sostenible. Retos del sector turístico ante la Agenda 2030. https://reds-sdsn.es/wp-content/uploads/2019/10/Gui\%CC\%81a-para-un-turismo-sostenible-REDS-RTI-web.pdf

Carlsen, J. y Dowling, R. (1998). Wine tourism marketing issues in Australia. International Journal of Wine Marketing, 10(3), 23 -32.

Charters, S. y Menival, D. (2011). Wine tourism in Champagne. Journal of Hospitality \& Tourism Research, 35(1), $102-118$.

Consell Regulador de la Denominació d’Origen Protegida. (2021). DO Empordà. https://www.doemporda.cat

Contreras, D. D. J., Thomé-Ortiz, H., Ortega, A. E. y Bordi, I. V. (2019). Trayectoria territorial de la región enológica de Querétaro, México (1970-2017): enoturismo y calidad territorial. Cuadernos Geográficos, 58(2), 240-261.

Contreras, D. D. J., Medina, F. X. y Thomé-Ortiz, H. (2020). Enogastronomía en la región vitivinícola de Querétaro, México: una perspectiva crítica sobre su patrimonialización. Quaderns de l'Institut Català d’Antropologia, 36(2), 257-277.

De Jesús-Contreras, D., Thomé-Ortiz, H. y Medina, F. X. (2020). Enoturismo y promoción del territorio. Análisis comparativo entre el nuevo y el viejo mundo del vino. PASOS Revista de Turismo y Patrimonio Cultural, 18(3), 457-471.

Du Rand, G. E. y Heath, E. (2006). Towards a framework for food tourism as an element of destination marketing. Current Issues in Tourism, 9(3), 206-234.

Ellis, A., Park, E., Kim, S. y Yeoman, I. (2018). What is food tourism? Tourism Management, 68, 250-263.

Fusté-Forné, F. (2015). El turisme gastronòmic: autenticitat i desenvolupament local en zones rurals. Documents d'Anàlisi Geogràfica, 61(2), 289-304.

Fusté-Forné, F. (2020). Savouring place: Cheese as a food tourism destination landmark. Journal of Place Management and Development, 13(2), 177-194.

Hall, C. M. y Sharples, L. (2003). The consumption of experiences or the experience of consumption? An introduction to the tourism of taste. En C. M. Hall, L. Sharples, R. Mitchell, N. Macionis y B. Cambourne (Eds.), Food Tourism Around the World: Development, Management and Markets (pp. 1-24). Butterworth-Heinemann.

Henderson, J. C. (2009). Food tourism reviewed. British Food Journal, 111(4), 317-326.

Hjalager, A. M. y Richards, G. (2003). Tourism and gastronomy. Routledge.

Jeambey, Z. (2016). Rutas Gastronómicas y Desarrollo local: un ensayo de conceptualización en Cataluña. Pasos Revista de Turismo y Patrimonio Cultural, 14(5), 1187-1198.

Kim, S. y Iwashita, C. (2016). Cooking identity and food tourism: The case of Japanese udon noodles. Tourism Recreation Research, 41(1), 89-100.

La Costa Brava promociona su enoturismo con una nueva campaña. (2020). La Vanguardia. https://www.lavanguardia.com/vida/ 20200508/481020937271/la-costa-brava-promociona-su-enoturismo-con-una-nueva-campana.html

Long, L. M. (2004). Culinary tourism. University Press of Kentucky.

López-Guzmán, T. y Sánchez-Cañizares, S. (2012). Gastronomy, tourism and destination differentiation: a case study in Spain. Review of Economics and Finance, 1, 63-72.

Medina, F. X. (2017). Reflexiones sobre el patrimonio y la alimentación desde las perspectivas cultural y turística. Anales de Antropología, 51(2), 106-113. 
Molleví, G. y Fusté-Forné, F. (2016). El turismo gastronómico, rutas turísticas y productos locales: el caso del vino y el queso en Cataluña. Geographicalia, 68, 25-47.

Okumus, B. (2020). Food tourism research: a perspective article. Tourism Review, 76(1), 38-42.

Organización Mundial del Turismo - OMT. (2010). Normas y sistemas de calidad en el turismo y su relación con la sostenibilidad y las leyes de turismo - La experiencia de las Américas. OMT.

Organización Mundial del Turismo - OMT. (2019). Definiciones de Turismo de la OMT. OMT.

Organización Mundial del Turismo - OMT. (2021). Desarrollo sostenible. https://www.unwto.org/es/desarrollo-sostenible

Organización Mundial del Turismo y Basque Culinary Center. (2019). Guía para el desarrollo del turismo gastronómico. OMT.

Organización Mundial del Turismo y Organización de los Estados Americanos. (2018). El turismo y los Objetivos de Desarrollo Sostenible - Buenas prácticas en las Américas. OMT.

Pérez, M. (2004). Manual del Turismo Sostenible. Ediciones Mundi-Prensa.

Romero, R. H. (2017). Rutas del Vino en España: enoturismo de calidad como motor de desarrollo sostenible. Ambienta: La Revista del Ministerio de Medio Ambiente, 118, 40-49.

Rousta, A. y Jamshidi, D. (2020). Food tourism value: Investigating the factors that influence tourists to revisit. Journal of Vacation Marketing, 26(1), 73-95.

Santos, V., Ramos, P., Sousa, B. y Valeri, M. (2021). Towards a framework for the global wine tourism system. Journal of Organizational Change Management, 1-13.

SECTUR. (2021). Portal de Turismo del Estado de Querétaro. https://www.queretaro.travel/es/regiones

Sidorenko, P. y Garrido, P. (2021). Comunicación y publicidad del turismo sostenible en la construcción de la marca ciudad. El caso de la certificación internacional ‘Biosphere Tourism’ en España. Revista Mediterránea de Comunicación, 12(1), 15-28.

Torres, A. M. (2016). Turismo Gastronómico Sostenible. Planificación de servicios, restaurantes, rutas, productos y destinos. Centro Empresarial Gastronómico Hotelero.

Velásquez, A. I. U. y Garlobo, E. R. (2020). La gastronomía, la cultura y el patrimonio para un turismo sostenible. Dilemas Contemporáneos: Educación, Política y Valores, 7(49), 1-15.

Wargenau, A. y Che, D. (2006). Wine tourism development and marketing strategies in Southwest Michigan. International Journal of Wine Marketing, 18(1), 45-60.

Marisa Morales Loya

Universitat de Girona, España.

Maestra en Turismo Cultural, Universidad de Girona, España. Licenciada en Administración Turística, Universidad Mondragón, México. Dedicada a la creación de proyectos sociales con enfoque en desarrollo sostenible. Investiga temáticas relacionadas al turismo gastronómico.

marisamoralesloya@outlook.com

ORCID: https://orcid.org/0000-0002-0431-8900

Francesc Fusté-Forné

Universitat de Girona, España.

Doctor en Turismo, Universidad de Girona, España. Doctor en Comunicación, Universidad Ramon Llull, España. Docente del Departamento de Empresa de la Universidad de Girona, España. Sus investigaciones analizan el patrimonio gastronómico y rural desde las perspectivas de marketing y turismo. francesc.fusteforne@udg.edu

ORCID: https://orcid.org/0000-0002-3800-9284 